\title{
The NEXT experiment to search for the neutrinoless double beta decay of $\mathrm{Xe}-136$
}

\author{
F. Monrabal on behalf of the NEXT Collaboration* \\ University of Texas at Arlington \\ 701 S. Nedderman Drive, Arlington, Texas, 76019 USA \\ E-mail: francesc.monrabalcapilla@uta.edu
}

The NEXT collaboration is building a $100 \mathrm{~kg}$ scale detector for neutrinoless double beta decay searches. The NEXT detector is a gaseous Time Projection Chamber (TPC) operating with xenon enriched at $90 \%$ in the Xe-136 isotope. Phase-I of the NEXT-100 detector, NEXT-White (NEW), is currently operating. With about half of the NEXT-100 linear dimensions (about $10 \mathrm{~kg}$ of xenon), NEW has the right size for demonstrating and fully understanding the different technological solutions to be implemented in NEXT-100, while keeping the number of sensors at a reasonable level. Furthermore, NEW is the first NEXT detector that is built with highly radio pure materials and that it will be operating underground at the Laboratorio Subterráneo de Canfranc (LSC). Its operation will permit a first in-situ measurement of the backgrounds to be expected in NEXT-100.

38th International Conference on High Energy Physics

3-10 August 2016

Chicago, USA

${ }^{*}$ Speaker. 


\section{NEXT technology}

The Neutrino Experiment with a Xenon TPC (NEXT) $)^{1}$ is a $\beta \beta 0 v$ experiment, using highpressure (15 bar) xenon (enriched at $90 \%$ in ${ }^{136} \mathrm{Xe}$ ) gas TPC with electroluminescent (EL) amplification of the ionisation signal.

Figure 1 shows the principle of operation of an High Pressure Xenon Electroluminescent (HPXe-EL) TPC. The detection process involves the use of the prompt scintillation light from the gas as start-of-event time, and the drift of the ionisation charge to the anode by means of an electric field $(\sim 0.3 \mathrm{kV} / \mathrm{cm}$ at 15 bar) where secondary EL scintillation is produced in a region with a field of $\sim 20 \mathrm{kV} / \mathrm{cm}$ at 15 bar. The detection of EL light provides an energy measurement using photomultipliers (PMTs) located behind the cathode (the energy plane) as well as tracking through its detection a few mm away from production at the anode, via a dense array of silicon photomultipliers (the tracking plane).

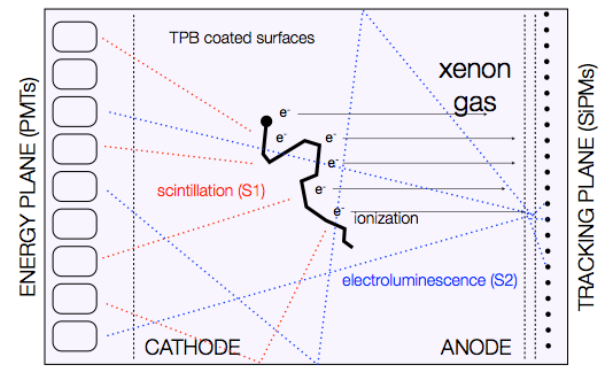

\subsection{R\&D}

Figure 1: Principle of operation of an HPXe-EL TPC.

The NEXT Collaboration has been operating two prototypes to demonstrate the HPXe-EL technology, NEXT-DEMO and NEXT-DBDM. NEXT-DEMO is equipped with an energy plane made of 19 Hamamatsu R7378A PMTs and a tracking plane made of 256 Hamamatsu SiPMs. The detector has been operating successfully for more than four years and has demonstrated: (a) excellent operational stability, with no leaks and very few sparks; (b) good energy resolution; (c) excellent electron drift lifetime, of the order of $20 \mathrm{~ms}[1,2,3]$; (d) track reconstruction with PMTs and with SiPMs coated with TPB and a first demonstration of the rejection power of the topological signature (Fig. 2 top) [4].

NEXT-DBDM, had a shorter drift region but with an aspect ratio (ratio diameter to length) similar to that of NEXT-100. The device has been used to perform detailed energy resolution studies, as well as studies to characterise neutrons in an HPXE-EL. NEXT-DBDM achieves an energy resolution of $1 \% \mathrm{FWHM}$ at $660 \mathrm{keV}$ and $15 \mathrm{bar}$, which extrapolates to $0.5 \%$ at $Q_{\beta \beta}$ (Fig. 2 bottom) [5].

\section{NEW}

The NEXT-White detector (NEW) is a radiopure High Pressure xenon Gas asymmetric TPC operating in EL mode at pressures between 10 and 15 bar at the Canfranc Underground Laboratory (LSC).

\footnotetext{
${ }^{1}$ http://next.ific.uv.es/next/
} 


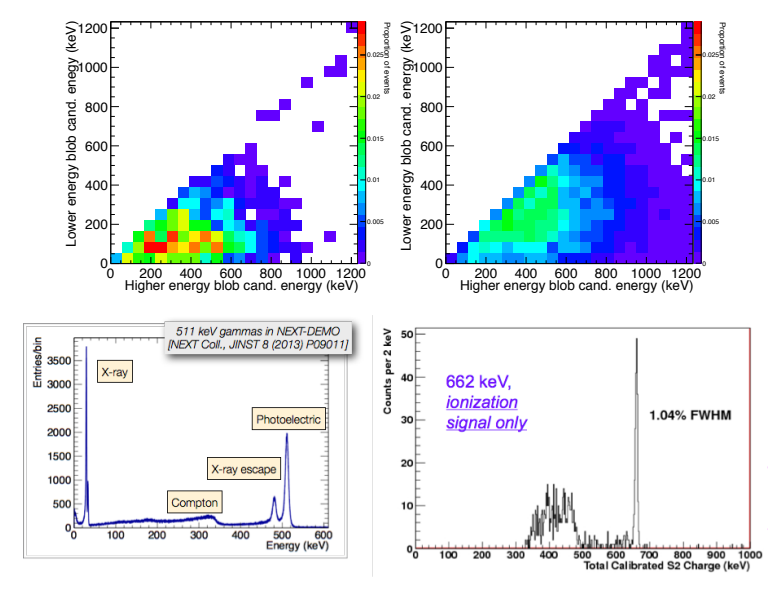

Figure 2: Top left: Energy distribution at the end-points of the tracks coming from ${ }^{22} \mathrm{Na}$ decay (single electrons). Top right: Distribution coming from the ${ }^{208} \mathrm{Tl}$ decay (pair produciton) for $2 \mathrm{~cm}$ radius blob (regions with higher density of ionized electrons) candidates. Bottom left: the full energy spectrum measured for electrons of $511 \mathrm{keV}$ in the NEXT-DEMO detector. Bottom right: Energy spectrum near the photoelectric peak for $662 \mathrm{keV}$ electrons in NEXT-DBDM. The resolution at $662 \mathrm{keV}$ is $1 \%$ FWHM (0.5\% FWHM at $Q_{\beta \beta}$ ). The resolution extrapolated from $511 \mathrm{keV}$ is $0.7 \%$.

The detector is equiped with 12 R11410 Hamamatsu PMTs (Fig. 3 left) $10 \mathrm{~cm}$ behind the cathode that allow for the detection of the prompt primary scintillation signal (S1) that is used as $a t_{0}$ of the event and gives the $z$ coordinate of the event. The PMTs are also responsible of the energy measurement by reading the secondary scintillation signal (S2) with high precision. Since the PMTs can not operate at high pressure, they are separated from the active volume by a $12 \mathrm{~cm}$ copper plate and operated at vacuum. The PMTs view the active volume through sapphire windows coated with Tetraphenyl Butadyene (TPB). In the opposite end of the detector, right behind the amplification region, a plane with 1792 SiPMs SensL series-C (Fig. 3 right) is distributed at a $1 \mathrm{~cm}$ pitch. The space in between SiPMs is covered by a thin $(2 \mathrm{~mm})$ teflon layer that will increase the total anode reflectivity and allows for a better light collection by the PMTs. The SiPMs permit a topological reconstruction of the events.

The NEW field cage creates a homogeneous field of $300 \mathrm{~V} / \mathrm{cm}$ in the drift volume and a field in the EL region of 2-3 kV/cm/bar. It has been fabricated using High density Poliethylene (HDPE) as a supporting material for the copper ring that create the drift field in the active volume (Fig. 3 left). The field cage support was produced by machining the grooves for the rings and the different details of the field cage from a single compact piece of HDPE. The copper rings are connected with $0.5 \mathrm{G} \Omega$ resistors. The buffer region (space between the cathode and the PMTs windows) used to degrade the cathode voltage consists on a serie of grooves that prevent charge movement through the surface. The EL region consists in a gate mesh built with stainless steel almost 100\% transparent to electrons and $\sim 95 \%$ transparent to photons. The anode is a fused silica plate coated with Indium Tin Oxyde in one side used to have a good countour condition definition and with TPB in the other side used to shift the VUV light emmited by the xenon to blue light where our sensors are much more efficient. The high voltage feedthroughs (HVFT) need to hold up to $20 \mathrm{kV}$ in the gate and $50 \mathrm{kV}$ in the cathode. The design of both is based in the design shown by H. Wang in [6] 
(Fig. 3 right). Finally a light tube is installed inside the field cage. The light tube is a teflon tube $10 \mathrm{~mm}$ thick coated with TPB designed to shift the VUV light to blue and to increase the teflon reflectivity.

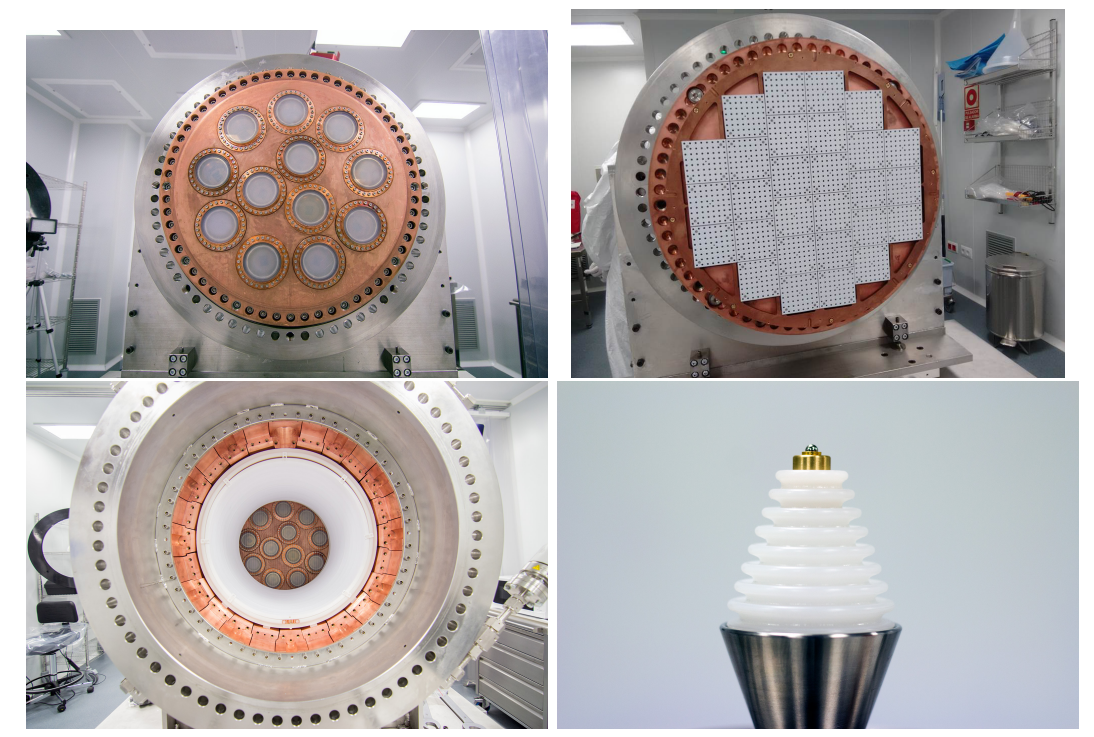

Figure 3: Top left: View of the full field cage installed including the reflector tube. Top right: Detail of the cathode HVFT with the conical shape with grooves and a spring connection at the end. Bottom left: View of the full field cage installed including the reflector tube. Bottom right: Detail of the cathode HVFT with the conical shape with grooves and a spring connection at the end.

\section{NEXT-100}

The next step for the collaboration is to build a $100 \mathrm{~kg}$ scale detector to be competitive with the current detectors in this scale and with an improved background rate. The design of NEXT-100 will be an upgrade to a large scale of the NEW detector. The active volume of the detector is a cylinder of approximately $1.15 \mathrm{~m}^{3}$ than can hold about $100 \mathrm{~kg}$ of xenon gas at 15 bar. It is surrounded by an open-ended high-density polyethylene (HDPE) cylindric shell, $2.5 \mathrm{~cm}$ thick, $148 \mathrm{~cm}$ long and $107.5 \mathrm{~cm}$ in diameter. The drift and amplification field will be similar to those used in NEW and that implies a necessary upgrade for the HVFT and the EL region design.

The energy and tracking plane use the same technology than the NEW detector but being a larger detector NEXT-100 will use 60 PMTs and near 7000 SiPMs.

\section{Background and sensitivity}

The relevance of any potential background source in NEXT depends on its probability to generate a signal-like track in the active volume of the detector with energy around the $Q$ value of ${ }^{136} \mathrm{Xe}$. In principle, charged particles (muons, betas, etc.) entering the detector can be eliminated with essentially perfect efficiency defining a small veto region (of a few centimetres) around the boundaries of the active volume. Confined tracks generated by external neutral particles (such as high-energy gamma rays) or by internal contamination in the xenon gas can be suppressed taking advantage of both the excellent energy resolution of the detector and the topological signature. 
Natural radioactivity in detector materials and surroundings is, as in most other $\beta \beta 0 v$-decay experiments, the main source of background in NEXT. In particular, the hypothetical $\beta \beta 0 \mathrm{v}$ peak of ${ }^{136} \mathrm{Xe}\left(Q_{\beta \beta}=2458.1 \pm 0.3 \mathrm{keV}\right)$ lies in between the photo-peaks of the high-energy gammas emitted after the $\beta$ decays of ${ }^{214} \mathrm{Bi}$ and ${ }^{208} \mathrm{Tl}$, intermediate products of the uranium and thorium series, respectively.

The contribution of each detector subsystem to the overall background rate of NEXT-100 is shown in Fig. 4.
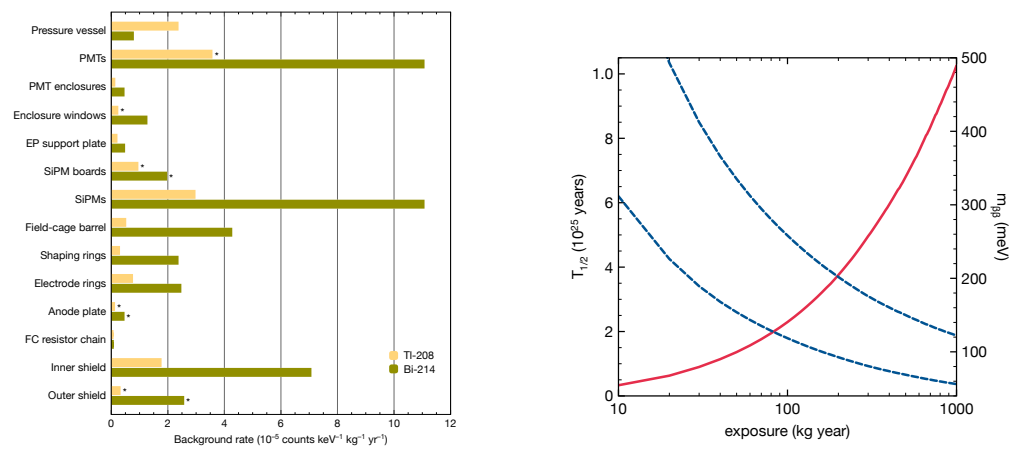

Figure 4: Right: Contribution to the background rate of NEXT-100 of the different detector subsystems considered in our background model. An asterisk (*) next to a bar indicates that the contribution corresponds to a positive measurement of the activity of the material. Left: Sensitivity (at $90 \% \mathrm{CL}$ ) to the $\beta \beta 0 v$-decay half-life (red. solid curve) and the corresponding effective Majorana neutrino mass sensitivity (blue, dashed curves) for the largest and smallest Nuclear Matrix Elements calculations in terms of the exposure. Plots from [7]

The expected background rate after cuts is at most $4 \cdot 10^{-4}$ counts $/ \mathrm{keV} / \mathrm{kg} / \mathrm{year}$. It is around 1 event per year for a $100 \mathrm{~kg}$ detector. That translates into an expected sensitivity of $6 \cdot 10^{25}$ years to the neutrinoless double beta decay process after three years of effective running MartinAlbo:2015rhw that will explore the Majorana mass of the neutrino in the $90-180 \mathrm{meV}$ region.

\section{Future improvements}

In order to build a tonne scale detector an extra effort on reducing the background rate needs to be done. The objective is to reach better than 1 event per year in the whole detector. Here I show some of the possible improvements that can be applied in a gaseous HPXE TPC.

\subsection{Improve effect of diffusion}

The topology signal is limited by the effect of the electron diffusion during the drift. This diffussion, with an rms of $10 \mathrm{~mm}$ after 1 meter of drift, can be improved using gaseous mixtures to the level of 2-3 $\mathrm{mm} \mathrm{rms}$ [8]. In parallel, improvements in the reconstruction of the energy deposition in the active volume and event selection are being developed [9].

\subsection{Tag Barium Ion}

The ultimate solution to the background problem will be the capability to tag the daughter ion produced in the decay. In this situation the only background contribution will be due to the finite 
resolution of the detector, in the case of a detector with very good energy resolution as NEXT it will be negligible. In the case of Xe-136 the daughter is a $\mathrm{Ba}++$ ion that can be tagged using a novel technique known as Single Molecule Fluorescence Imaging (SMFI) [10]. This technique has been succesfully applied in biology and the NEXT Collaboration is trying to apply it to a xenon gaseous detector.

\section{Acknowledgments}

The NEXT Collaboration acknowledges support from the following agencies and institutions: the European Research Council (ERC) under the Advanced Grant 339787-NEXT; the Ministerio de Economía y Competitividad of Spain under grants CONSOLIDER-Ingenio 2010 CSD20080037 (CUP), FIS2014-53371-C04 and the Severo Ochoa Program SEV-2014-0398; the Portuguese FCT and FEDER through the program COMPETE, project PTDC/FIS/103860/2008; the U.S. Department of Energy under contracts number DE-AC02-07CH11359 (Fermi National Accelerator Laboratory) and DE-FG02-13ER42020 (Texas A\&M); and the University of Texas at Arlington.

\section{References}

[1] V. Álvarez, et al., Initial results of NEXT-DEMO, a large-scale prototype of the NEXT-100 experiment, JINST 8 (2013) P04002. arXiv:1211.4838.

[2] V. Álvarez, et al., Operation and first results of the NEXT-DEMO prototype using a silicon photomultiplier tracking array, JINST 8 (2013) P09011. arXiv:1306.0471.

[3] V. Álvarez, et al., Ionization and scintillation response of high-pressure xenon gas to alpha particles, JINST 8 (2013) P05025. arXiv:1211.4508, doi:10.1088/1748-0221/8/05/P05025.

[4] P. Ferrario, et al., First proof of topological signature in the high pressure xenon gas TPC with electroluminescence amplification for the NEXT experiment, JHEP 01 (2016) 104. arXiv:1507.05902, doi:10.1007/JHEP01(2016)104.

[5] V. Alvarez, et al., Near-Intrinsic Energy Resolution for 30 to $662 \mathrm{keV}$ Gamma Rays in a High Pressure Xenon Electroluminescent TPC, Nucl. Instrum. Meth. A708 (2013) 101-114. arXiv:1211.4474, doi:10.1016/j.nima.2012.12.123.

[6] B. Rebel, C. Hall, E. Bernard, C. H. Faham, T. M. Ito, B. Lundberg, M. Messina, F. Monrabal, S. P. Pereverzev, F. Resnati, P. C. Rowson, M. Soderberg, T. Strauss, A. Tomas, J. Va'vra, H. Wang, High voltage in noble liquids for high energy physics, Journal of Instrumentation 9 (08) (2014) T08004. URL http://stacks.iop.org/1748-0221/9/i=08/a=T08004

[7] J. MartÃ $n$-Albo, et al., Sensitivity of NEXT-100 to neutrinoless double beta decay, JHEP 05 (2016) 159. arXiv:1511.09246, doi:10.1007/JHEP05(2016)159.

[8] C. D. R. Azevedo, L. M. P. Fernandes, E. D. C. Freitas, D. Gonzalez-Diaz, F. Monrabal, C. M. B. Monteiro, J. M. F. dos Santos, J. F. C. A. Veloso, J. J. Gomez-Cadenas, An homeopathic cure to pure Xenon large diffusion, JINST 11 (02) (2016) C02007. arXiv:1511.07189, doi:10.1088/1748-0221/11/02/C02007.

[9] J. Renner, et al., Background rejection in NEXT using deep neural networksarXiv:1609.06202.

[10] D. R. Nygren, Detecting the barium daughter in ${ }^{136} \mathrm{Xe} 0-v \beta \beta$ decay using single-molecule fluorescence imaging techniques, J. Phys. Conf. Ser. 650 (1) (2015) 012002. doi:10.1088/1742-6596/650/1/012002. 